\title{
Prueba de discriminación auditiva de sonidos del habla. Prueba SH
}

\author{
Ángela María Abad, Manuel García, Manuel Peralbo \\ Grupo de Investigación en Psicología del desarrollo y del Aprendizaje Escolar \\ Departamento de Psicoloxía Evolutiva e da Educación, Universidade da Coruña
}

\begin{abstract}
Resumen
En el presente trabajo se muestran los resultados preliminares de una prueba diseñada específicamente para evaluar la discriminación auditiva de sonidos del habla (SH) en niños y niñas de los primeros cursos de la educación primaria y los últimos cursos de educación infantil. La Prueba SH se ha aplicado a una muestra de 40 niños y niñas elegidos aleatoriamente entre varios grupos de $2^{\circ}$ de Educación Primaria.
\end{abstract}

Palabras clave: Discriminación auditiva, construcción de pruebas, educación primaria

\section{Introducción}

El desarrollo de un correcto sistema de comunicación oral está directamente relacionado con la discriminación auditiva de los sonidos del habla, puesto que si un oyente no es capaza de distinguir las diferencias entre los distintos sonidos que componen nuestra lengua no va a ser capaza de representarlos correctamente ( en forma de sonidos o palabras). Hablar de los sonidos que se producen oralmente para comunicarnos implica hablar de fonemas. Los fonemas son unidades de análisis lingüísticos carentes de

\section{Método}

\section{Participantes}

La muestra evaluada está compuesta por 40 niños y niñas elegidos aleatoriamente entre varios grupos de $2^{\circ}$ curso de Educación Primaria (21 niñas y 19 niños) de un colegio público del área metropolitana de A Coruña. Los alumnos seleccionados procedían de clase media o media-baja y están escolarizados desde los 3 años. Las características socioeconómicas de los sujetos tienen un perfil homogéneo debido al centro educativo seleccionado. Ninguno de los niños había recibido previamente instrucciones de uso de las pruebas. Todos los niños y niñas presentaban lenguaje oral, estaban en periodo lector, no presentaban ninguna alteración a nivel orgánico ni funcional de los órganos fonoarticulatorios $y$ no presentaban alteraciones sensoriales (exceptuando los alumnos que usaban gafas) o cognitivas.

\section{Instrumentos}

La prueba de discriminación auditiva SH consta de tres apartados o subpruebas. Una primera subprueba (prueba de habituación) de adaptación a distintos sonidos del habla, en el que el sujeto debe de estar atento a un cambio auditivo. Cuando se detecta el cambio el sujeto tiene que pulsar una tecla para indicar significado que constituyen la abstracción de un sonido y poseen la propiedad de distinguir significados.

Que un sonido no tenga significado por si mismo, pero nos ayude a distinguir que palabra está produciendo un hablante (rana o rama) y que significado podemos o debemos de darle, para poder hacernos una representación de dicho significado, es primordial para comprender el entorno que nos rodea.

A pesar de que los fonemas no tienen significado en un entorno aislado, si tienen rasgos distintivos que nos ayudan a diferenciarlos de forma auditiva y con ello poder articularlos correctamente.

En nuestra lengua existen fonemas que se diferencian en tan solo un rasgo (punto de articulación, modo de articulación o acción de las cuerdas) y es esta característica diferenciadora la que tenemos que ser capaces de discernir para poder saber que palabra está emitiendo, articulando un hablante.

En este estudio hemos aislado sonidos de nuestra lengua, los hemos producido de forma individual, los hemos producido dentro de palabras representadas de forma escrita y los hemos producido dentro de palabras emitidas oralmente, con la finalidad de elaborar un "aprueba screening” que nos ayude a detectar la existencia de dificultades auditivas en la percepción del habla que interfieran en el desarrollo comunicativo. que ha escuchado el cambio. En la segunda subprueba (prueba de dibujos) el sujeto tiene que seleccionar entre tres dibujos (previamente nombrados ) cuál de ellos contiene un fonema que se ha emitido de forma aislada. En la tercera subprueba ( prueba de palabras) el sujeto tiene que seleccionar entre tres palabras (previamente nombradas) cuál de ellas contiene el fonema que se ha emitido en aislado.

En la primera subpruebas el cambio que se produce después de la habituación a un sonido determinado y que debe ser detectado por el sujeto, es un cambio hacia un sonido el cual solo difiere del primero en un rasgos distintivo.

En la segunda subprueba, en la que el sujeto tiene que encontrar en que dibujo se encuentra el fonema a discriminar, se le presentan dibujos que en su nominación son par mínimo del fonema a discriminar. Uno de los dibujos contiene un fonema el cual solo difiere en un rasgo distintivo del fonema inicialmente emitido. El otro dibujo que se presenta en la misma pantalla que los anteriores, se distingue del dibujo considerado como correcto en que el fonema que cambia contiene más de un rasgo distintivo en relación al fonema emitido como correcto.

La tercera subprueba tiene un desarrollo igual que la segunda subprueba, lo único que cambia es el estímulo 
visual, en vez de dibujos se presentan palabras de tres en tres.

La subprueba de sonidos está elaborada para evaluar a niños con y sin lectura, la subprueba de dibujos está pensadas para niños sin lectura o pre-lectores, mientras que en la subprueba de palabras es necesario que el niño esté en un periodo lector.

Las tres subpruebas están informatizadas, lo cual nos permite obtener datos de error y acierto, tiempos de reacción y tipo de ayudas que se le presentan a los sujetos evaluados.

Además cada participante ha sido evaluado con la prueba EDAF que evalúa la discriminación auditiva y fonológica

\section{Procedimiento}

Cada sujeto de la muestra fue evaluado de marea individual, en una habitación aislada, en la cual el sonido ambiente nunca fue superior al emitido por la prueba. Los niños y niñas en ningún momento tienen contacto visual con la boca del examinador a modo de ayuda visual para discriminar los sonidos emitidos, durante la realización de las tareas de la prueba. Las evaluaciones se realizaron entre los meses de abril, mayo y junio, y se llevaron a cabo en periodos de 45-60 minutos, durante 1 o 2 días, en función de los niveles de cansancio y atención de los sujetos. Debido a la novedad de la tarea, que una parte importante estaba informatizada y que ello conllevaba la interrupción de la jornada habitual de clases, todos los alumnos participaron de forma voluntaria y activa. Antes del inicio de cada prueba se realizaba un contacto oral y visual positivo con el alumno. La evaluación se realizó en horario y periodo escolar, respetando los recreos estipulados por el centro educativo. El evaluador había sido presentado previamente al grupo de alumnos y a los profesores del centro. Los profesores tenían información previa de las características de la investigación.

\section{Resultados}

En estos resultados solo representamos una parte representativa de los datos obtenidos de subpruebas del EDAF de discriminación fonológica y de logotomas, así como los resultados obtenidos en subpruebas de habituación al sonido y en la subprueba de discriminación fonológica de palabras, de la Prueba SH.

Tabla 1.

EDAF. Discriminación de logotomas

\begin{tabular}{l|c|c|c|}
\hline Logotomas & Aciertos & Errores & Fonemas \\
\hline 1.ma pa pa & 38 & 2 & $\mathrm{M}-\mathrm{P}$ \\
2.ma ba ba & 38 & 2 & $\mathrm{M}-\mathrm{B}$ \\
3.fa fa za & 28 & 12 & $\mathrm{~F}-\mathrm{C} / \mathrm{Z}$ \\
4.za za za & 36 & 2 & $\mathrm{C} / \mathrm{Z}$ \\
5.ta da da & 35 & 5 & $\mathrm{~T}-\mathrm{D}$ \\
6.da da da & 39 & 1 & $\mathrm{D}$ \\
7.ta ka ka & 35 & 5 & $\mathrm{~T}-\mathrm{K}$ \\
8.ta ta ta & 37 & 3 & $\mathrm{~T}$ \\
9.cha ka cha & 36 & 4 & $\mathrm{CH}-\mathrm{K}$ \\
10.ta cha ta & 39 & 1 & T-CH \\
11.cha cha cha & 40 & 0 & $\mathrm{CH}$
\end{tabular}

\begin{tabular}{llcc} 
12.za sa sa & 39 & 1 & Z/C-S \\
13.sa sa sa & 40 & 0 & $\mathrm{~S}$ \\
14.ja ja ka & 38 & 2 & $\mathrm{~J}-\mathrm{K}$ \\
15.ka ka ka & 39 & 1 & $\mathrm{~K}$ \\
16.ga ja ja ja & 39 & 1 & $\mathrm{G}-\mathrm{J}$ \\
17.ka ga ga & 31 & 9 & $\mathrm{~K}-\mathrm{G}$ \\
18.ma na na & 39 & 1 & $\mathrm{M}-\mathrm{N}$ \\
19.na na na & 40 & 0 & $\mathrm{~N}$ \\
20.na ña na & 40 & 0 & $\mathrm{~N}-\mathrm{N}$ \\
21. la la ra & 40 & 0 & $\mathrm{~L}-\mathrm{R}$ \\
22. la la la & 40 & 0 & $\mathrm{~L}$ \\
23.da ra da & 38 & 2 & $\mathrm{D}-\mathrm{R}$ \\
24.ra ra ra & 25 & 15 & $\mathrm{R}$ \\
25.rra rra la & 38 & 2 & $\mathrm{RR}-\mathrm{L}$ \\
26.rra rra rra & 36 & 4 & $\mathrm{RR}$ \\
27. ya ya rra & 39 & 1 & $\mathrm{Y}-\mathrm{RR}$ \\
28.ya ya ya & 40 & 0 & $\mathrm{Y}$ \\
\hline
\end{tabular}

En las tablas 1 y 2 están recogidos los datos obtenidos de forma directa en dos de las subpruebas de la prueba EDAF, discriminación auditiva de logotomas y discriminación auditiva de fonemas. Los datos se exponen sin tener en cuenta los índices de error que han sido utilizados para la creación de las puntuaciones de dicha prueba. Esto quiere decir que no se han tenido en cuenta errores de producción oral que se emiten en las pruebas y que dan lugar a respuesta de "falso-acierto", como es caso del ítem 24 de la tabla 1. En la tabla 2 la palabras subrayada en cada ítem es la emitida por la prueba como palabras a identificar entre dos dibujos.

Tabla 2.

EDAF. Discriminación de fonemas.

\begin{tabular}{l|c|c|c|}
\hline D. de fonemas & Aciertos & Errores & Par mínimo \\
\hline 1. Mesa-Pesa & 40 & 0 & M-P \\
2.Marco-Barco & 40 & 0 & M-B \\
3.Bolo-Polo & 40 & 0 & B-P \\
4.Cubo-Tubo & 40 & 0 & K-T \\
5.Carro-Tarro & 40 & 0 & K-T \\
6.Vaca-Bata & 40 & 0 & K-T \\
7.Torre-Corre & 40 & 0 & T-K \\
8.Casa-Caza & 39 & 1 & S-Z/C \\
9.Pollo-Polo & 40 & 0 & LL-L \\
10.Ola-Olla & 40 & 0 & L-LL \\
11.Rana-Rama & 39 & 1 & N-M \\
12.Mono-Moño & 40 & 0 & N-Ñ \\
13.Gota-Jota & 31 & 9 & G-J \\
14.Nata-Nada & 40 & 0 & T-D \\
15. Reja-Teja & 38 & 2 & RR-T \\
16.Caja-Paja & 40 & 0 & K-P \\
17.Cuna-Luna & 40 & 0 & K-L \\
18.Rata-Lata & 40 & 0 & RR-L \\
19.Cazo-Lazo & 40 & 0 & K-L \\
20.Rana-Lana & 40 & 0 & RR-L \\
21. Leña-Peña & 40 & 0 & L-P \\
22.Gafas-Gatas & 40 & 0 & F-T \\
23.Sopa-Copa & 40 & 0 & S-K \\
24.Boda-Bola & 40 & 0 & D-L \\
25. CocheNoche & 40 & 0 & K-N
\end{tabular}




\begin{tabular}{lllc} 
26.Mocho-Moco & 40 & 0 & CH-K \\
27. Hucha-Uva & 40 & 0 & CH-B/V \\
28.Pelo-Perro & 40 & 0 & L-RR \\
29.Ropa-Copa & 40 & 0 & RR-K \\
30.Rama-Cama & 40 & 0 & RR-K \\
31.Tierra-Sierra & 40 & 0 & T-S \\
32. Foca-Roca & 40 & 0 & F-RR \\
33. Sopa-Ropa & 40 & 0 & S-RR \\
34. Alto-Salto & 40 & 0 & Omisión S \\
35.Lata-Ata & 40 & 0 & Omisión L \\
36. Rosa-Osa & 40 & 0 & Omisión \\
37. Boca-Oca & 40 & 0 & Omisión \\
38.Ola-Cola & 40 & 0 & Omisión K \\
39.Tiza-Tia & 38 & 2 & Omisión \\
40.Calle-Cae & 39 & 1 & Omisión \\
41.Casa-Caja & 39 & 1 & S-J \\
\hline
\end{tabular}

Los sonidos que se emiten en esta subrueba de habituación y que están representados en la primera columna de la Tabla 3 tienen un duración aproximada de entre 15 y 17 segundos producidos de forma continua hasta que se produce el primer cambio. En cada ítem se producen dos cambios auditivos y cada ítem tiene una duración completa de entre 32 y 35 segundos. La respuesta que se recoge en esta subprueba tiene cuatro variables tres son incorrectas y una correcta; que el sujeto no perciba ninguno de los dos cambios que se producen, que discrimine el sonido después del segundo cambio sonoro, que se produzca una respuesta antes de que exista el cambio sonoro real y una respuesta correcta. La discriminación de pares mínimos en la que más errores, de forma general, se produjeron por parte de los participantes ha sido el cambio sonoro del fonema $\mathrm{M}$ al fonema $\mathrm{N}$.

Tabla 3.

Prueba SH. Habituación al sonido

\begin{tabular}{c|c|c|c|c|}
\hline Sonidos & $\begin{array}{c}\text { No } \\
\text { Pulsó }\end{array}$ & $\begin{array}{c}\text { Pulsa en } \\
2^{\circ} \text { cambio } \\
\text { sonoro }\end{array}$ & $\begin{array}{c}\text { Se precipitó/ } \\
\text { notó cambio }\end{array}$ & Correcto \\
\hline S-F-S & 0 & 1 & 0 & 39 \\
C-S-C & 0 & 0 & 0 & 40 \\
M-N-M & 7 & 2 & 0 & 31 \\
T-P-T & 2 & 0 & 1 & 37 \\
B-D-B & 1 & 0 & 1 & 38 \\
K-T-K & 1 & 0 & 0 & 39 \\
D-G-D & 1 & 0 & 0 & 39 \\
P-K-P & 1 & 0 & 0 & 39 \\
M-B-M & 1 & 0 & 0 & 39 \\
R-L-R & 1 & 0 & 1 & 38 \\
N-L-N & 0 & 0 & 0 & 40 \\
K-G-K & 1 & 0 & 0 & 39 \\
T-D-T & 0 & 0 & 0 & 40 \\
P-B-P & 0 & 0 & 1 & 39 \\
G-B-G & 1 & 0 & 0 & 39 \\
F-C-F & 1 & 0 & 0 & 39 \\
\hline
\end{tabular}

La tabla 4 representa datos recogidos de cada uno de los participantes en la Prueba $\mathrm{SH}$, relacionados con el ítem en el cual tienen que discriminar el fonema K. En esta tabla 4 se observa un elevado número de aciertos en la discriminación del par mínimo K-G, un 67,5\% de los sujetos reconocieron el sonido y fueron capaces de ubicarlo dentro de la palabra correcta. Un 32,5\% emitieron una respuesta errónea. Dentro de esta respuesta tenemos dos tipos de errores posibles que el sujeto confunda el sonido con un par mínimo que tenga una diferencia de un rasgo distintivo (palabra que contenía el sonido K) con el sonido correcto o que tenga más de un rasgo distintivo (palabra que contenía el sonido $\mathrm{S}$.

Tabla 4.

Prueba SH. Subprueba de palabras.

\begin{tabular}{|c|c|c|c|}
\hline Fonemas K-G-S & Aciertos & Errores & Tipo \\
\hline Sujeto 1 & 1 & 0 & \\
\hline Sujeto 2 & 1 & 0 & \\
\hline Sujeto 3 & 1 & 0 & \\
\hline Sujeto 4 & 0 & 1 & S \\
\hline Sujeto 5 & 1 & 0 & \\
\hline Sujeto 6 & 0 & 1 & G \\
\hline Sujeto 7 & 0 & 1 & G \\
\hline Sujeto 8 & 0 & 1 & G \\
\hline Sujeto 9 & 0 & 1 & S \\
\hline Sujeto 10 & 1 & 0 & \\
\hline Sujeto 11 & 1 & 0 & \\
\hline Sujeto 12 & 0 & 1 & G \\
\hline Sujeto 13 & 1 & 0 & \\
\hline Sujeto 14 & 1 & 0 & \\
\hline Sujeto 15 & 0 & 1 & G \\
\hline Sujeto 16 & 1 & 0 & \\
\hline Sujeto 17 & 1 & 0 & \\
\hline Sujeto 18 & 1 & 0 & \\
\hline Sujeto 19 & 0 & 1 & G \\
\hline Sujeto 20 & 1 & 0 & \\
\hline Sujeto 21 & 0 & 1 & S \\
\hline Sujeto 22 & 1 & 0 & \\
\hline Sujeto 23 & 1 & 0 & \\
\hline Sujeto 24 & 0 & 1 & S \\
\hline Sujeto 25 & 1 & 0 & \\
\hline Sujeto 26 & 0 & 1 & G \\
\hline Sujeto 27 & 1 & 0 & \\
\hline Sujeto 28 & 1 & 0 & \\
\hline Sujeto 29 & 1 & 0 & \\
\hline Sujeto 30 & 1 & 0 & \\
\hline Sujeto 31 & 1 & 0 & \\
\hline Sujeto 32 & 1 & 0 & \\
\hline Sujeto 33 & 1 & 0 & \\
\hline Sujeto 34 & 0 & 1 & S \\
\hline Sujeto 35 & 1 & 0 & \\
\hline Sujeto 36 & 1 & 0 & \\
\hline
\end{tabular}




\begin{tabular}{llll} 
Sujeto 37 & 1 & 0 & \\
Sujeto 38 & 0 & 1 & S \\
Sujeto 39 & 1 & 0 & \\
Sujeto 40 & 1 & 0 & \\
\hline Total & 27 & 13 & - \\
\hline
\end{tabular}

\section{Discusión}

La intención de la elaboración de la Prueba SH es obtener una prueba screening para detectar dificultades en la discriminación de fonemas del habla. En la comparativa que se va a realizar con otras pruebas ya baremadas vamos a tener en cuenta los fonemas que difieren en un solo rasgo distintivo, puesto que cuantos menos rasgos distintivos existan entre dos sonidos emitidos oralmente más dificultad existe en su discriminación auditiva. En estos momentos de la investigación solo podemos ofrecer resultados relacionados con la observación y no concluyentes. Hemos decidido ampliar la muestra del grupo de edad analizado y a otros grupos de edad para poder discutir la fiabilidad y validez de la Prueba SH. Lo que si podemos decir a través de la observación directa de la aplicación de la prueba es que al emitir los fonemas en aislado nos encontramos con que los oyentes lectores presentan dificultad para reconocer los fonemas de su lengua y de imitarlos oralmente, cosa que sucede en menos ocasiones cuando ese fonema va acompañado de una vocal (silaba directa o inversa) o se presenta dentro de una palabra. Esta diferencia se puede observar directamente en los datos directos que se presentan en las tablas 1, 2, 3 y 4 . En las tablas 1 y 2 se producen menos errores auditivos que en las tablas 3 y 4 . Los datos de las tablas 1 y 2 referencias la emisión de fonemas que van acompañados de otros sonidos vocalicos y consonánticos dando lugar a la emisión de silabas o palabras, mientras que en las tablas 3 y 4 los sonidos se emiten en aislado.

\section{Referencias}

Alonso-Cortés, A. (2002), Lingüística, Madrid: Ediciones Cátedra.

Brancal, M. (1998), EDAF Evaluación de la discriminación auditiva y fonológica, , Lebón

Cervera, J. \& Ygual, A. (2001). Estrategias para la evaluación de la percepción de los rasgos fonológicos. EDENTIA, Estudios y propuestas de educación. $N^{o}$ 15, 57-62.

Clemente, A. R. (1995). Desarrollo del lenguaje. Manual para profesionales de la intervención en ambientes educativos. Barcelona: Octoedro Universidad.

Marrero, V. (2001). Fonética y fonología de la lengua española. Fonética Perceptiva-ADDENDA. Madrid: UNED.

Mayor, M. A., Zubiauz , B. (2011), LOLEVA

Pavez, M., Maggiolo, M., Peñazola, C. \& Coloma, C. (2009). Desarrollo fonológico en niños de 3 a 6 años: incidencia de la edad, el genero y el nivel socioeconómico. RLA. Revista de lingüística teórica y aplicada. $N^{\circ} 47$ (2), 89-109.

Villegas, F. (2004). Manual de logopedia: evaluación e intervención en las dificultades fonológicas. Madrid: Ediciones Pirámide.

Zenker, F., Suárez, M., Marro, S. \& Barajas del Prat, J. J. (2007). La evaluación del procesamiento auditivo central: el test de dígitos dicóticos. Revista de Logopedia, Foniatría y Audiología. № 2, 74-85. 